\title{
Survival Effect of Tube Feeding in Patients with Advanced Dementia and Comorbidities
}

\author{
Efraim Jaul ${ }^{*}$, Raz Levin ${ }^{2}$, Jacob Menczel $^{3}$ \\ ${ }^{1}$ Skilled Geriatric Nursing Department, Herzog Memorial Hospital, The Hebrew University and Hadassah \\ Medical School, Jerusalem, Israel \\ ${ }^{2}$ Department of the Neurobiology, Herzog Memorial Hospital, The Hebrew University and Hadassah Medical \\ School, Jerusalem, Israel \\ ${ }^{3}$ Department of Geriatrics, Institute of Osteoporosis, Herzog Memorial Hospital, The Hebrew University and \\ Hadassah Medical School, Jerusalem, Israel \\ Email: ${ }^{\text {jaul@zahav.net.il }}$
}

Received 19 February 2014; revised 18 March 2014; accepted 17 April 2014

Copyright (C) 2014 by authors and Scientific Research Publishing Inc.

This work is licensed under the Creative Commons Attribution International License (CC BY). http://creativecommons.org/licenses/by/4.0/

(c) (i) Open Access

\section{Abstract}

Patients suffering from advanced dementia who have difficulties in eating and swallowing are often tube fed (TF). They suffer, on most occasions, from additional diseases. The effect of tube feeding in advanced dementia patients is controversial and is largely discussed in the medical literature. The purpose of the present study, is to report the effect of tube feeding on the survival time in patients with advanced dementia who suffered from additional diseases. One hundred and forty nine patients with Functional Assessment Stage of Alzheimer Disease (FAST) stage 5 $\geq$, hospitalized in skilled geriatric nursing department between July 2008 and December 2011 were included in the study. Oral and tub feeding was decided following the evaluation by speech therapists at admission. $99(66.5 \%)$ of the patients were tube fed and $50(33.5 \%)$ received oral feeding at admission. The mean age was $80 \pm 11$. Sixty four percent of the patients were admitted from general hospitals. Comorbidities were significantly higher in the tube fed group, median survival time was 164 days. The median survival time of the OF group was 78 days $\left(\chi^{2}=0.94 ; p=0.33\right)$. Prevalence of tube feeding increased significantly from $53.85 \%$ to $90.9 \%$ in patients with 3 risk factors such as pressure ulcers, low Hgb and low BMI. The survival time of the tube fed patients was longer compared to patients who were orally fed. Tube feeding in patients with dementia continues to be controversial, and additional researchable data are helpful in the decision to insert a tube.

*Corresponding author. 


\section{Keywords}

\section{Advance Dementia, Tube Feeding, Survival Time}

\section{Introduction}

Elderly people with advanced dementia frequently suffer from eating and swallowing difficulties, and as a result, the liquid and caloric intake is very low, and as to be expected, weight is lost [1]. The benefits and disadvantages accompanying the feeding problems in patients with dementia are thoroughly discussed [2]. The decision to insert a tube for feeding is usually made due to the incapability of the patient to eat or to drink, and with the consent of the family.

Mitchell et al., followed 323 residents with advanced dementia in nursing homes for 18 months, indicating 85.8\% had eating problem. The six month mortality rate associated with eating problem was 38.6\% [3].

Finucane et al., have not found that tube feeding (TF) in patients with advance dementia prevents aspiration pneumonia, prolongs survival time or improvement of pressure ulcers [4]. The American Geriatric Society (AGS) recently published five principles for physicians and patients should question regarding tube feeding decisions. The first recommendation preferred oral assisted feeding (OF) in patients with advance dementia [5]. A Cochrane systematic review reported by Sampson et al. resulted in insufficient evidence to suggest enteral tube feeding is beneficial [6]. Teno et al. reported that percutaneous endoscopy gastroscopy (PEG) feeding even increased the risk of pressure ulcers in nursing home patients with advanced dementia [7]. Adverse effects, such as aspiration pneumonia does occur in TF [8]. Although most of the studies found no benefits for TF, in advanced dementia patients, one third of them in USA nursing homes are tube fed [9]. A pilot study determined TF in a skilled nursing department significantly increased the survival time in comparison with those fed orally [10]. The different results may be explained by the severity of the associated illnesses in patients with advanced dementia.

Dwolatzky et al. showed improvement of survival time in a group of non-acute patients with long-term enteral feeding [11].

There is a constant increase of dementia associated with eating problems. More knowledge of the course of the disease is helpful in determining the right decision, especially in issues and concerns regarding tube feeding.

The aim of the present study is to report the effect of TF on the survival of patients with advanced dementia also suffering from severe diseases; and to compare these with similar orally fed (OF) patients. The effect of specific risk factors on the survival period of TF patients was also examined.

\section{Methods}

Data were collected from the files of patients hospitalized in the skilled geriatric nursing department of the Herzog Hospital in Jerusalem between July 1, 2008, and December 31, 2011. Admission criteria to the Skilled Geriatric Nursing Department included patients bound to bed and having one or more of the following complications: extensive and deep pressure ulcers (grade 3 - 4); terminal cancer requiring palliative care; renal failure requiring hemodialysis; oxygen dependence; non-invasive ventilation and tracheostomy.

The inclusion criteria of the hospitalized patients were the Reisberg's Functional Assessment Staging of Alzheimer Disease (FAST) phase 5 - 7 [12]. The patients were admitted with TF while others were orally fed.

During the first week of hospitalization all patients underwent an evaluation of the feeding method (OF or TF), and the necessity for tube feeding. The evaluation was done by the speech therapist and assisted by the other professional staff of the department. If patients were able to swallow the tube was withdrawn and individuals were orally fed. Patients who were not able to swallow or to drink continued to be tube fed. A skin examination and the Norton scale were performed by the nurse. Physical examination including the Glasgow Coma Scale (GCS) was performed by a physician [13]. The dietitian assessed weight, BMI, and recommended the caloric and protein intake. The muscle strength and the cognitive state were evaluated by the physiotherapist and occupational therapist. The socioeconomic states of the patient's family were assessed by the social worker. Routine blood examinations (CBC and SMAC) were performed. 
A structured questionnaire was completed for each patient by a physician upon admission. The data was collected from the medical, nursing, social and nutritional files and included the socio-demographic characteristics, comorbidities, use of urinary catheter, tube feeding, tracheostomy, as well as pressure ulcer assessment. Mental state assessment was difficult to perform in most cases, due to lack of verbal cooperation. Therefore, the Functional Assessment Staging (FAST), the Glasgow Coma Scale (GCS) and the Norton Scale were used. According to the FAST staging instrument, ascertaining of cognitive levels are: Stage 1: No Cognitive Decline, Stage 2: Very Mild Cognitive Decline, Stage 3: Mild Cognitive Decline, Stage 4: Moderate Cognitive Decline, Stage 5: Moderately Severe Cognitive Decline, Stage 6: Severe Cognitive Decline, Stage 7: Very Severe Cognitive Decline. The Glasgow Coma Scale (GCS) measures consciousness (score ranging 3 - 15, with a score of 5 - 9 indicating vegetative state, whereas $\geq 10$ indicates alertness. The Norton scale score assesses five risk-based items: sensory perception, physical, mental condition, incontinence, activities and mobility. The score range from 4 24 points and a score of $\geq 14$ is considered a high risk for developing PU's [14].

Descriptive statistics, by feeding method (TF/OF) at study initiation are presented for all study participants. Logistic regression models were used to estimate the Odds Ratio (OR) and 95\% confidence intervals (CI) for TF vs OF by study covariates. A multivariate logistic regression model was fitted for a select group of variables, which were chosen according to clinical standards at baseline. Variables entered included age, gender, Norton scale, Albumin, Hgb, BMI, FAST, GCS, urinary catheter. All variables were entered at the first stage and backward elimination was used to remove non-significant factors, the criterion for removal from the model was a p-value above 0.10 .

The association between feeding method and survival time was assessed by the Kaplan-Meier model.

All data were analyzed with SPSS software (version 17.0; Chicago, IL). All statistical tests were two-sided. A p value below 0.05 was considered significant.

The study was approved by the Helsinki Committee Herzog Hospital.

\section{Results}

One hundred forty nine patients were included in the study: 99 (66.5\%) were with TF and 50 (33.5\%) with OF at admission. The mean age of the entire group was $80 \pm 11$. The age of the TF patients was not significantly different from OF group $(79.23 \pm 11.19$ vs $80.36 \pm 10.04)$.

The characteristics of the two groups were compared by univariant analyses and are shown in Table 1.

Sixty four percent of the patients were admitted from general hospitals, $19.5 \%$ from home and $15 \%$ from nursing care institutions. No significant differences in TF rates was observed between the patients admitted form the different places.

The underlying medical conditions were significantly higher in the TF group, such as: cerebrovascular accidents (CVA), chronic renal failure (CRF) and lung diseases (Table 1). Parkinson's disease, diabetes mellitus, ischemic heart disease (IHD) and peripheral vascular disease (PVD) were not significantly different between the two groups.

The mean Functional Assessment Staging of Alzheimer Disease (FAST) was significantly higher in the TF group (6.54 \pm 0.6 vs. $6.02 \pm 0.5, \mathrm{p}<0.001)$. The mean Glasgow Coma Scale (GCS) (10.9 \pm 2.99 vs. $13.8 \pm 1.64$, $\mathrm{p}<0.001)$ and the total Norton score $(8.75 \pm 2.2$ vs $11.04 \pm 2.5 ; \mathrm{p}<0.001)$ were significantly lower in the TF patients compare to the OF patients.

Statistically significant higher prevalence of pressure ulcers (75\% vs $25 \%$ ), tracheostomies (91\% vs. 9\%) and urinary catheters (72\% vs. 28\%) were found in the TF patients.

The total lymphocyte count (TLC; 1727 vs 1448) and the hemoglobin levels (Hgb; 10.8 vs 10.23) were statistically higher in the TF group. In contrast, BMI was significantly higher in the OF group (22.9 vs 25.9) (Table 1). Serum albumin, cholesterol and total protein were not different in the two groups.

The median survival time of patients with TF was 164 days and that of the OF group was 78 days $\left(\chi^{2}=0.94\right.$; $\mathrm{p}=0.33$ ) (Figure 1).

In the TF group patients with pressure ulcers, low Hgb and low BMI revealed higher, yet not statistically significant, median survival time in comparison with OF group (Table 2).

Risk factors (i.e. pressure ulcers, low Hgb and low BMI) significantly increased the prevalence of TF from $53.85 \%$ with no risk factors to $90.9 \%$ with 3 risk factors (Table 3 ). 
Table 1. Characteristics of study population.

\begin{tabular}{|c|c|c|c|c|c|}
\hline Characteristics & $\begin{array}{l}\text { Tube Feeding } \\
\qquad(\mathrm{n}=99)\end{array}$ & $\begin{array}{l}\text { Non Tube Feeding } \\
\qquad(\mathrm{n}=50)\end{array}$ & OR & $95 \%$ CL & p-value \\
\hline Age (yr) (mean \pm SD) & $79.23 \pm 11.19$ & $80.36 \pm 10.04$ & 0.99 & $0.96-1.02$ & 0.55 \\
\hline Male & $48(67.6 \%)$ & $23(33 \%)$ & \multirow[b]{2}{*}{1.10} & \multirow[b]{2}{*}{$0.56-2.18$} & \multirow[b]{2}{*}{0.77} \\
\hline Female & $51(65.4 \%)$ & 27 (34.6\%) & & & \\
\hline Transfer from general hospital & $65(31.3 \%)$ & $30(68.8)$ & 0.97 & $0.36-2.64$ & 0.70 \\
\hline Transfer from home & $17(58.6 \%)$ & $12(41.4 \%)$ & 0.64 & $0.27-1.51$ & 0.35 \\
\hline Transfer from Nursing home & $15(68.2 \%)$ & 7 (31.8\%) & 1.08 & $0.41-2.86$ & 0.87 \\
\hline Cerebrovascular accident & $46(79.3 \%)$ & $12(20.7 \%)$ & \multirow[b]{2}{*}{0.36} & \multirow[b]{2}{*}{$0.17-0.78$} & \multirow[b]{2}{*}{$0.009^{*}$} \\
\hline No Cerebro-vascular accident & $53(58.2 \%)$ & $38(41.8 \%)$ & & & \\
\hline Lung Disease & $61(73.5 \%)$ & $22(26.5 \%)$ & \multirow[b]{2}{*}{0.49} & \multirow[b]{2}{*}{$0.25-0.98$} & \multirow{2}{*}{$0.04^{*}$} \\
\hline No Lung Disease & 38 (57.6\%) & 28 (42.4\%) & & & \\
\hline Chronic Renal failure & $30(55.5)$ & $24(44.5)$ & \multirow[b]{2}{*}{2.12} & \multirow[b]{2}{*}{$1.05-4.28$} & \multirow[b]{2}{*}{$0.03^{*}$} \\
\hline No Chronic Renal failure & $69(72.6 \%)$ & $26(27.4 \%)$ & & & \\
\hline $\begin{array}{l}\text { Functional Assessment Staging of Alzheimer } \\
\text { Disease (FAST) total, Mean }\end{array}$ & $6.54 \pm 0.6$ & $6.02 \pm 0.5$ & 3.72 & $2.04-6.80$ & $0.001^{*}$ \\
\hline Glasgow Coma Scale (GCS) total, Mean & $10.9 \pm 2.99$ & $13.8 \pm 1.64$ & 0.55 & $0.44-0.69$ & $0.001^{*}$ \\
\hline Norton Scale total score, Mean & $8.75 \pm 2.2$ & $11.04 \pm 2.5$ & 0.68 & $0.57-0.80$ & $0.001^{*}$ \\
\hline $\begin{array}{l}\text { Urinary catheter } \\
\text { No Urinary catheter }\end{array}$ & $\begin{array}{c}68(72) \\
31(56.4 \%)\end{array}$ & $\begin{array}{c}26(28) \\
24(43.6 \%)\end{array}$ & 0.49 & $0.25-0.99$ & $0.05^{*}$ \\
\hline No tracheostomy & 79 (62.2\%) & 48 (37.8\%) & 0.16 & $0.04-0.74$ & $0.02^{*}$ \\
\hline $\begin{array}{c}\text { Pressure Ulcer } \\
\text { No Pressure Ulcer }\end{array}$ & $26(49.1 \%)$ & 27 (50.9\%) & 0.35 & $0.17-0.70$ & $0.003^{*}$ \\
\hline Total Lymphocyte Count Mean & $1727 \pm 720$ & $1448 \pm 659$ & 1.0 & $1.0-1.0$ & $0.03^{*}$ \\
\hline $\mathrm{Hb}$ (gr\%) Mean & $10.80 \pm 1.58$ & $10.23 \pm 1.30$ & 1.30 & $1.02-1.65$ & $0.03^{*}$ \\
\hline BMI Mean & $22.87 \pm 5.16$ & $25.93 \pm 6.46$ & 0.91 & $0.85-0.98$ & $0.008^{*}$ \\
\hline
\end{tabular}

*Significant $\mathrm{p}<0.05$ values are in (\%). Global Deterioration Scale (GDS), score range: 1 (no disability) - 7 (severe dementia); Glasgow Coma Scale (GCS), total range 3 - 15; score 5 - 9 indicates vegetative state, >10 alert. Norton Scale, score range 4 - 24.

Table 2. The median survival time of tube and oral fed patients, with pressure ulcers, low Hgb and low BMI population (KM results).

\begin{tabular}{ccc}
\hline Risk factors & Median survival of TF patient (days) & Median survival of OF patient (days) \\
\hline Pressure ulcers & 96 & 68 \\
Low Hgb & 96 & 37 \\
Low BMI $<22$ & 172 & 96.5 \\
\hline
\end{tabular}


Table 3. The distribution of the study population by numbers of risk factors.

\begin{tabular}{cccc}
\hline \multirow{2}{*}{ Number of risk factors } & \multicolumn{2}{c}{ Number of patients (\%) } & Total \\
\cline { 2 - 3 } & Oral feeding group & Tube feeding group & 26 \\
No risk factors & $12(46.15 \%)$ & $14(53.85 \%)$ & 49 \\
Single risk factors & $23(46.94 \%)$ & $26(53.06 \%)$ & 52 \\
Two risk factors & $13(25 \%)$ & $39(75 \%)$ & 22 \\
Three risk factors & $2(9.09 \%)$ & $20(90.91 \%)$ & \\
\hline
\end{tabular}

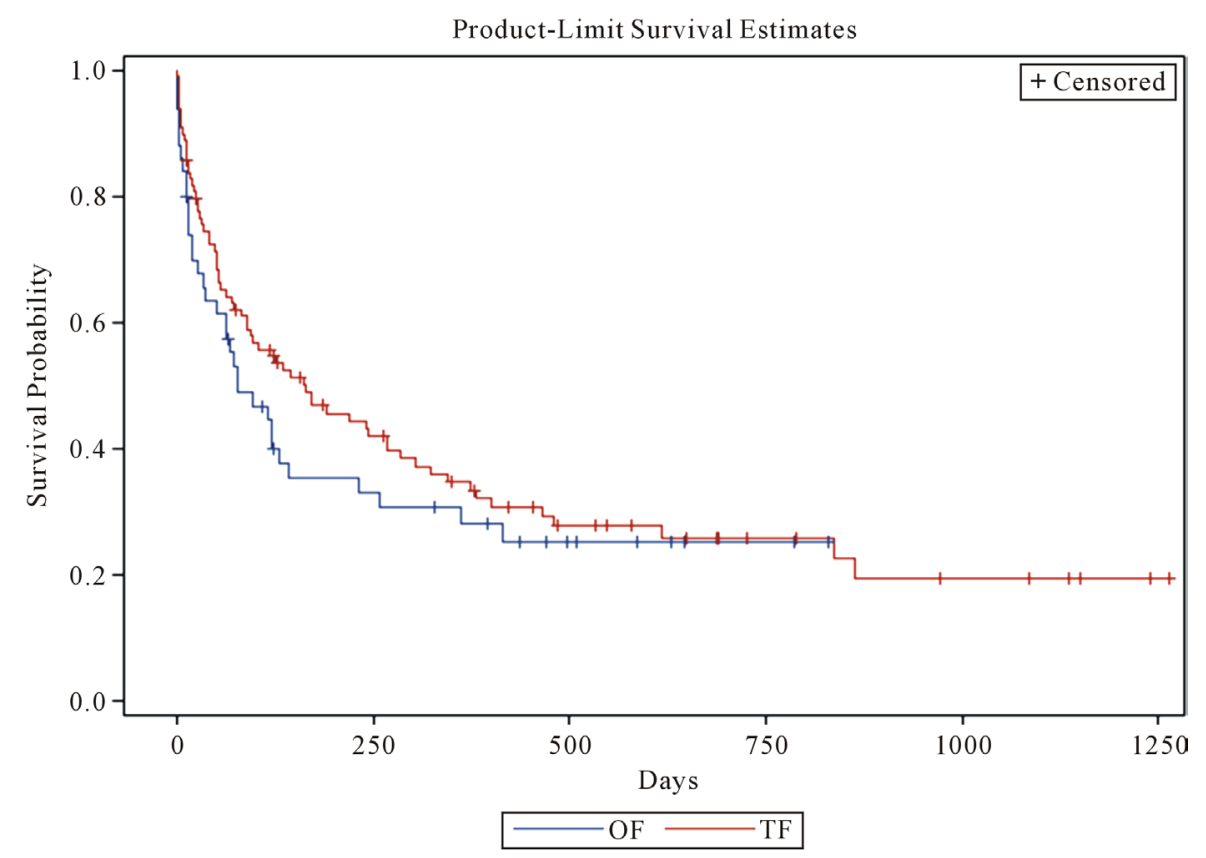

Figure 1. Kaplan-Meier median survival of patient with feeding tube and oral feeding.

\section{Discussion}

This study relates to advanced dementia patients also suffering, from other severe diseases including cerebrovascular accidents (CVA), chronic renal failure (CRF) and lung diseases. Due to these additional diseases patients were referred to the Department of Skilled Nursing.

The tube fed group suffered from more complications including pressure ulcers, low Hgb level, use of urinary catheters and tracheostomies as compared to the oral fed patients. The cognitive and functional parameters had more pathologic values in the tube fed patients such as; higher FAST, lower GCS and lower Norton scores. Lower Glasgow Coma Scale (GCS) reflected the patient's low consciousness. This severe condition was the result of the persistent vegetative state following a traumatic event or an extensive ischemic brain accident. These patients are, in fact, unable to eat by mouth at all, due to lack of alertness as well as neurological impairment. The decision for oral assisted feeding does not arise in these cases. In contrast a patient, who was severely demented, although suffering from a large pressure ulcer in the sacrum, was orally assisted fed by the family or the staff. Families are enlisted to feed the patient or to recruit a private care taker.

The main result of the study is that the survival time of the TF patients was longer, yet not statistically significant. TF was beneficial in patients with specific risk factors, the median survival times such as low Hgb, low BMI and pressure ulcers, was higher in the TF group versus OF group.

The indication to insert a tube is simply to supply the patients with basic nutrition-calories and fluids-because there is no other alternative way to provide feeding. The patient reported suffered from aspiration pneumonia, malnutrition, functional decline and pressure ulcers, even before the insertion of a feeding tube. The ethical 
question was of survival versus ending life.

The study reflects that patients with advanced dementia suffer, in addition to decline in cognitive function and lower alertness, from multiple comorbidities such as: renal, lung and cardiovascular diseases, low BMI, low Hgb, pressure ulcers and the need of urinary catheter. These risk factors interact and complicate treatment of the patient's overall health condition.

The study limitations include the study's setting; conducted in a skilled nursing department rather than in the community or in nursing homes. It is associated with a selection bias towards sicker patients with higher comorbidities. Our control group is composed of patients who met department admission criteria. Therefore our results may not be generalizable to community or nursing homes patients.

\section{Conclusion}

The advanced dementia patients described in the study were unable to eat and to drink, suffered from additional diseases, and had a clear indication for tube feeding. Recognizing the existence of comorbidities with advanced dementia patients, the study concluded that the median survival period in the TF group was longer as compared to the OF patients.

\section{Conflict of Interest}

The authors (Efraim Jaul, Raz Levin and Jacob Menczel) declare that there is no financial support or relationship that may pose conflicts of interest and no potential conflicts of interest were disclosed.

\section{References}

[1] Vitale, C.A., Monteleoni, C., Burke, L., Della Frazier, R. and Volicer, L. (2009) Strategies for Improving Care for Patients with Advanced Dementia and Eating Problems. Annals of Long Term Care, 17, 32-39.

[2] Gillick, M.R. (2000) Rethinking the Role of Tube Feeding in Patients with Advanced Dementia. New England Journal of Medicine, 342, 206-209. http://dx.doi.org/10.1056/NEJM200001203420312

[3] Mitchell, S.L., Teno, J.M., Kiely, D.K., Shaffer, M.L., Jones, R.N., Prigerson, H.G., Volicer, L., Givens, J.L. and Hamel, M.B. (2009) The Clinical Course of Advanced Dementia. New England Journal of Medicine, 361, 1529-1538. http://dx.doi.org/10.1056/NEJMoa0902234

[4] Finucane, T.E., Christmas, C. and Travis, K. (1999) Tube Feeding in Patients with Advanced Dementia. A Review of the Evidence. The Journal of the American Medical Association, 282, 1365-1370. http://dx.doi.org/10.1001/jama.282.14.1365

[5] American Geriatric Society Workgroup (2013) Identifies Five Things That Healthcare Providers and Patients Should Question. Journal of American Geriatric Society, 61, 622-631. http://dx.doi.org/10.1111/jgs.12226

[6] Sampson, E.L., Candy, B. and Jones, L. (2009) Enteral Tube Feeding for Older People with Advanced Dementia. Cochrane Database Systematic Reviews, 2, CD007209. http://dx.doi.org/10.1002/14651858.CD007209.pub2

[7] Teno, J.M., Gozalo, P., Mitchell, S.L., et al. (2012) Feeding Tubes and the Prevention or Healing of Pressure Ulcers. Archives of Internal Medicine, 172, 697-701. http://dx.doi.org/10.1001/archinternmed.2012.1200

[8] Finucane, T.E. and Bynum, J.P. (1996) Use of Tube Feeding to Prevent Aspiration Pneumonia. Lancet, 348, 14211424. http://dx.doi.org/10.1016/S0140-6736(96)03369-7

[9] Mitchell, S.L., Teno, J.M., Roy, J., et al. (2003) Clinical and Organizational Factors Associated with Feeding Tube Use among Nursing Home Residents with Advanced Cognitive Impairment. The Journal of the American Medical Association, 290, 73-80. http://dx.doi.org/10.1001/jama.290.1.41

[10] Jaul, E., Singer, P. and Calderon-Margalit, R. (2006) Tube Feeding in Demented Elderly with Severe Disabilities. Israel Medical Association Journal, 8, 870-874.

[11] Dwolatzky, T., Berezovski, S., Friedmann, R., Paz, J., Clarfield, A.M., Stessman, J., Hamburger, R., Jaul, E., Friedlander, Y., Rosin, A. and Sonnenblick, M. (2001) A Prospective Comparison of the Use of Nasogastric and Percutaneous Endoscopic Gastrostomy Tubes for Long-Term Enteral Feeding in Older People. Clinical Nutrition, 20, 535-540. http://dx.doi.org/10.1054/clnu.2001.0489

[12] Reisberg, B. (1998) Functional Assessment Staging (FAST). Psychopharmacology Bulletin, 24, 653-659.

[13] Teasdale, G. and Jennett, B. (1974) Assessment of Coma and Impaired Consciousness: Practical Scales. Lancet, 2, 81-84. http://dx.doi.org/10.1016/S0140-6736(74)91639-0

[14] Norton, D., McLaren, R. and Exton-Smith, A.N. (1962) An Investigation of Geriatric Nursing Problems in Hospital. Churchill Livingstone, London, 193-224. 